\title{
Talbot Lithography with soft elastomeric conformal phase masks
}

\author{
Rudy Desgarceaux ${ }^{1,2}$, Zhanna Santibayeva ${ }^{1}$, Francesco Pedaci ${ }^{1}$, and Benoît Charlot ${ }^{2}$. \\ ${ }^{1}$ Centre de Biochimie Structurale, CBS CNRS UMR5048, INSERM U1054, Montpellier, France \\ ${ }^{2}$ Institut d'Electronique et des Systèmes, IES, CNRS UMR 5214, Université de Montpellier, Montpellier, France
}

\begin{abstract}
We report a novel method to create 2D hierarchical nanopatterns with a simple PDMS scaffold made of a replica of a grating and a spacer. This technique takes advantage of the Talbot effect that is the creation of an interference pattern from a grating at a given distance normal to the grating surface. By combining a conformal soft mask made of PDMS and a spacer to separate the phase shift mask from the photoresist, we can take advantage of the interference pattern to imprint smaller structures.
\end{abstract}

\section{Introduction}

Periodic nanostructures found many applications in several fields such as photonics, for creating surface plasmon resonance (SPR) detectors, in material science for creating superhydrophobic or omniphobic surfaces but also in number of other applications in physics, optics, and even biophysics. The fabrication of such nanostructured requires however low cost fabrication processes able to pattern large surfaces. The creation of periodic nanostructures through a top down approach by means of conventional lithography techniques, e.g contact lithography with chromium masks or phase shift masks is limited by the minimal optical resolution and most of the time by the cost of advanced lithography equipments. Electron beam Lithography (EBL) that uses a scanning electron beam to expose specific photoresists can achieve higher resolution, down to tens of nanometers but at the expense of high cost equipments and long time exposure. Nano Imprint lithography (NIL) is a technique that allows creating high-resolution structures with high throughput [4]. However, it is a replication technique that provides no gain in terms of resolution with respect to the master used for the imprint. Interference lithography (IL) is a technique that uses interference of coherent lights to create periodic patterns; it is one of the most economical techniques to create such regular nanostructures over large ( $\left.\mathrm{cm}^{2}\right)$ surfaces. IL has been used to create one- and two-dimensional periodic patterns. The minimum period of the patterns available in IL is equal to $\lambda / 2 \mathrm{n}$, where $\lambda$ is the wavelength in vacuum and $\mathrm{n}$ is the refractive index of the medium.

Herein, we report a simple method to create diverse hierarchical 2D nanopatterns using high-order diffraction patterns that occurs when light passes through a transparent conformal mask made of PDMS that has been cast on an array of nano cylinders. This technique is close to phase shift lithography but here the masks are not made of an opaque layer of chromium on glass but is made of a fully transparent piece of PDMS that embeds an array of holes. These holes that are filled with air have an optical index that is different of the index of the PDMS thus creating and phase shift in the optical path an inducing diffraction patterns. It is this diffraction pattern that will be used to expose the photoresist that is placed in contact with the soft mask.

\section{Talbot effect.}

When a grating is illuminated with sufficiently coherent light (wavelength $\lambda$ ), self-images of the grating are observed at periodic distances $p$ away from the normal of the grating. These so-called Talbot images occur with a period of $\mathrm{p} 2 / \lambda$. At certain integer fractions of this period, the image frequency is a multiple of the grating frequency. This effect has been used to create high- resolution lithographic structures [5] and this technique is called the Displacement Talbot Lithography. The principle is to use a photomask that contains an array of holes and expose a substrate covered with a photoresist with a distance between the mask and the substrate, this distance being a fraction of the Talbot distance $Z=p 2 / \lambda$. With this it is possible to imprint regular arrays of structures that are smaller than the original array and with shapes and spacings that can be of interest for plasmonic resonances.

This paper will present a lithography technique that combines the use of a soft conformal mask and the Talbot displacement lithography method. Using a PDMS mask containing an array of holes in addition with a spacer made also in PDMS and with a thickness smaller than the Talbot period it is possible to imprint diffraction patterns smaller that the original array. This technique allows creating plasmonic structures with ease and efficiency using conventional lithography equipment.

\section{Experimental section}

The fabrication procedure starts with the replica molding of the first grating: a periodic structure of photoresist is made by LIL on a $2 \times 2$ silicon substrate and then etched with an inductively coupled plasma reactive ion etching (ICP RIE) reactor to produce an array of $1 \mu \mathrm{m}$ tall cylinders [2]. Next, a mixture of PDMS (DuPont Sylgard 184) and reticulating agent (10\% weigth) is poured over the nanocylinder array. The viscous preparation is then degas under vacuum and reticulated for 2 hours at $80^{\circ} \mathrm{C}$. The piece of hardened PDMS is then extracted from the positive master (cylinder array). Next a spacer is made by spincoating a thin layer of degazed PDMS on a silicon 
wafer at $6000 \mathrm{rpm}$ for $10 \mathrm{~min}$ and baked on a hotplate at $90^{\circ} \mathrm{C}$ for $20 \mathrm{mn}$. To measure the exact height of the spacer we use a profilometer (DEKTAK). The height of the deposition is an essential factor because it set the integer fractions of the Talbot period that we will be used in conformal mask. The deposition height is controled by the spin-coating time.

Both grating replica and spacer are then exposed to an oxygen plasma (Diener Nano) for $1 \mathrm{~min}$ at low power to activate the surfaces. These two parts are finally bonded together to build a Talbot soft conformal mask as can be seen in Figure 3 where we can observe the array of cylindrical holes and the spacer that has been partially removed for the photography. The exposure is made on a piece of silicon or any other material that is covered with a thin layer of photoresist. Any type of photoresist can be used, positive or negative, but it has to be thin regarding the pitch of the grating in order to avoid out of plane interferences. The exposition process is very fast and easy to use.

\section{Results and discussion}

As we can see in figure 4 and 5, the structures are easily reproduced with a direct contact between the PDMS stamp and the photoresist of the sample (quartz or Si...). Ether with positive or negative photoresist it is possible to replicate a periodic pattern in a small amount of time. In figure 4(a) we use a positive resist so we create an array of disk with the same pitch as the one on the soft mask. In figure 5 (a) it is the same pattern but with negative resist as a consequence we obtain an array of holes that can be further used for a lift off process.

Figure 5(b,c) shows the surface of a sample where photoresist has been exposed using a soft mask that contains a spacer to take advantage of the Talbot displacement effect. We can notice in the picture that another array is intercalated in between the original one, decreasing its pitch by a factor $\sqrt{2} 2$. Figure 5 (a) shows also a photoresist pattern made with this technique, and we can notice the inclusion of a set of 4 small structures in between the main holes having the same period as the original grating.

\section{Conclusions}

We present a simple and efficient technique that allows the creation of small sized periodic patterns from a soft elastomeric conformal mask made of pieces of PDMS. Using the Talbot effect and a construction of a PDMS grating and a spacer we can take advantage of the Talbot Displacement lithography technique to imprint arrays of periodic patterns that are smaller than the original array. These periodic patterns can be used also to create a phase shift mask and to decrease another time the pitch of the periodic pattern. 

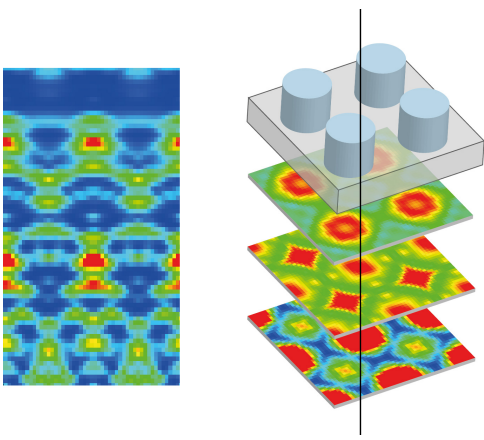

Figure 2. FDTD simulation of the Talbot effect. Light diffraction by a repeated pattern of cylindrical holes in a PDMS block. Intensity of light the normal direction of the grating and in the plane at several distances.

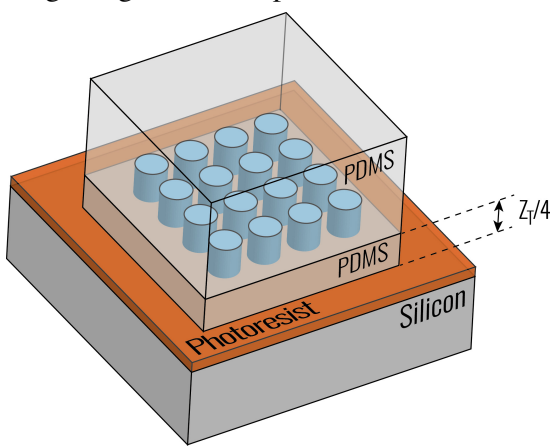

Figure 1. Schematic of the soft PDMS mask made of a network of cylindrical aperture and a spacer.

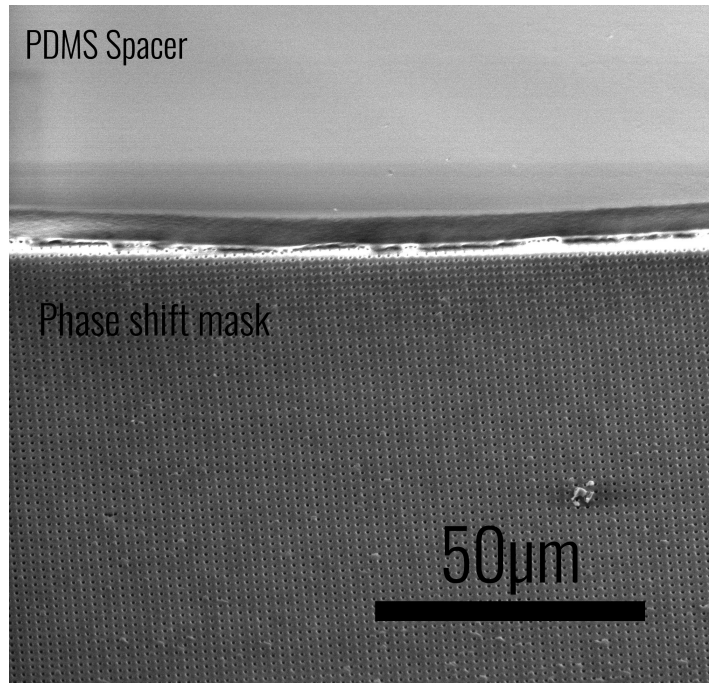

Figure 3. SEM image of the placement of a thin PDMS membrane acting as a spacer in front of the PDMS matrix.

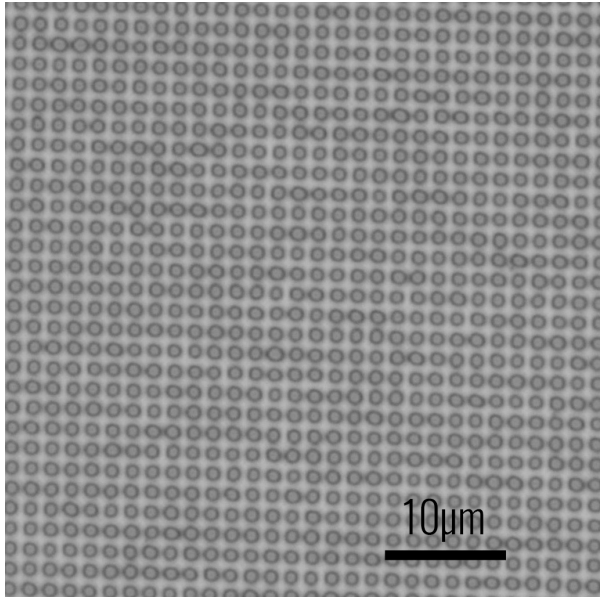

(a)

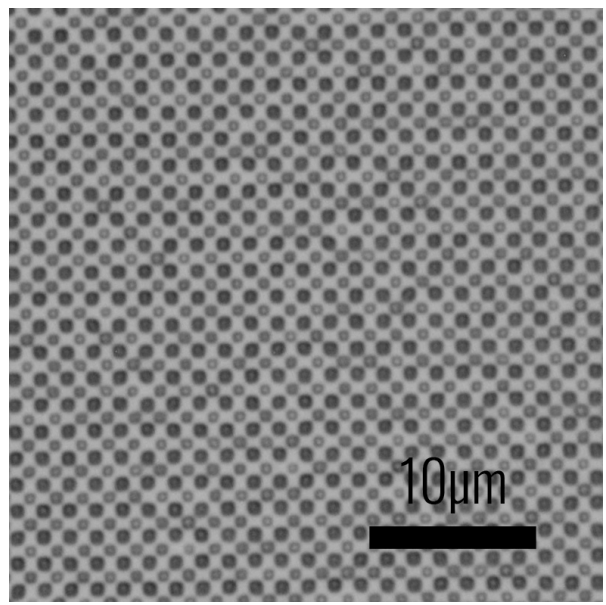

(b)

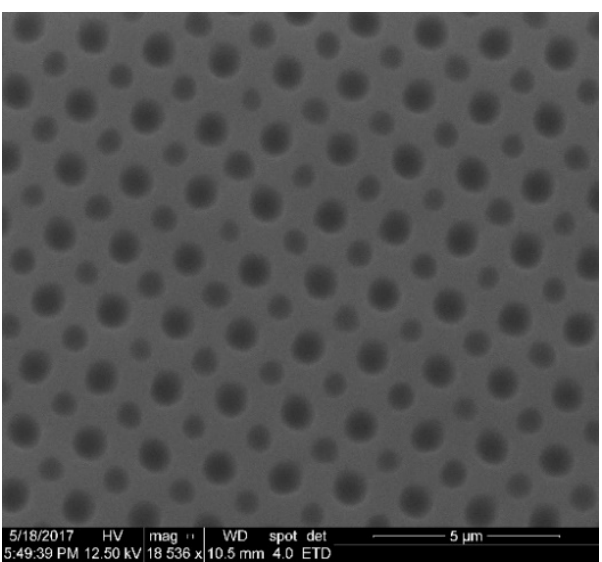

(c)

Figure 4. Patterns transferred into positive phototoresist by exposure through PDMS soft mask (A) and PDMS soft mask with a spacer $(B, C)$. We can notice the inclusion of a 2nd array of holes intercalated within the first one. 


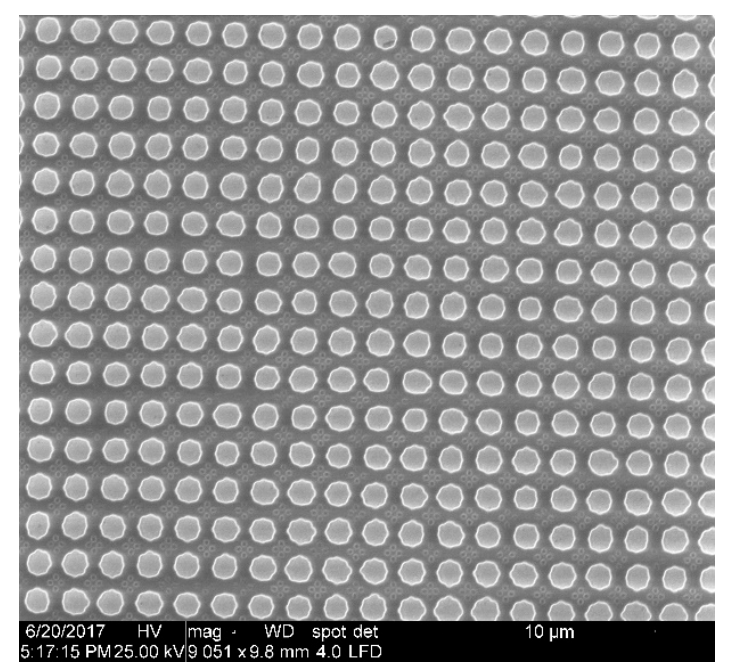

(a)

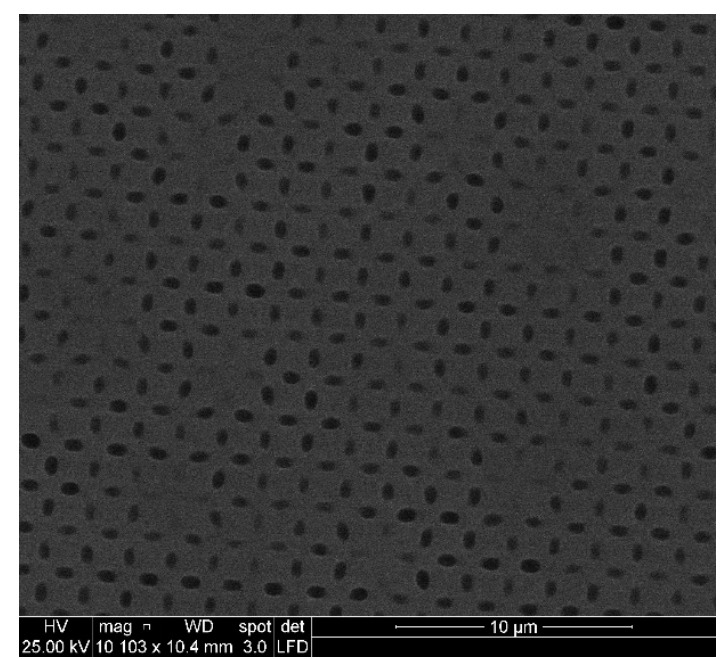

(b)

Figure 5. Pattern transferred into negative photoresist by exposure through PDMS soft mask with (A) and without spacer (B). A totally different pattern was created just by inserting a spacer between the phase shift mask and the substrate.

\section{References}

[1] H. Talbot, Philosophical Magazine Series 3, 1836; 9(56), 401-407.

[2] J.A. Rogers, K.E. Paul, R.J. Jackman, G. M. Whitesides, "Using an elastomeric phase mask for sub-100 nm photolithography in the optical near field," Appl. Phys. Lett., vol. 70, no. 20, (1997).

[3] Z.Santybayeva, A.Meghit, R.Desgarceaux, R.Teissier, F.Pichot, C. de Marin, B.Charlot, F.Pedaci "Fabrication of quartz micro-cylinders by laser interference lithography for angular optical tweezers", Journal of Micro/Nanolithography, MEMS, and MOEMS. 15(3), o34507 (2016).

[4] J.Henzie, M.H. Lee, T.W.Odom, "Multiscale patterning of plasmonic metamaterials" Nature nanotechnology 2, 549-554 (2007)

[5] C.P. Fucetola, A.A. Patel, E.E. Moon, Thomas B. O'Reilly, and Henry I. Smith "Coherent diffraction lithography: Periodic patterns via mask-based interference lithography"

J. Vac. Sci. Technol. B (27)6 (2009) 